\title{
Detection and genome analysis of human bocavirus 1-4 from hospitalized children with acute lower respiratory tract infection and symptoms of wheezing in Shanghai
}

\author{
JUN SHEN, QIRONG ZHU, MEI ZENG and HUI YU \\ Department of Infectious Diseases, Children's Hospital of Fudan University, Shanghai 201102, P.R. China
}

Received June 14, 2013; Accepted September 13, 2013

DOI: $10.3892 / \mathrm{ijmm} .2013 .1512$

\begin{abstract}
The aim of this study was to elucidate the clinical and molecular epidemiology of human bocavirus (HBoV)1-4 in hospitalized children in Shanghai suffering from acute lower respiratory tract infection with symptoms of wheezing. HBoV1-4 was detected by nested PCR from 275 nasopharyngeal secretion samples collected from hospitalized children. The HBoV-positive DNA sequences were aligned and phylogenetic trees were constructed. The detection rate of HBoV1 was $5.45 \%(15 / 275)$, which was second only to respiratory syncytial virus (RSV). HBoV1 was co-detected with other potential pathogens in most of the samples. No sample was HBoV2-4-positive. Homology analysis of the partial VPI/VP2, $N P 1$ and NS1 sequences revealed that these genes belonged to the same HBoV1 genotype. Furthermore, the phylogenetic analysis indicated that these epidemic strains clustered on one independent branch. Our results demonstrate that HBoV1 may be one of the common pathogens responsible for the hospitalization of children with acute lower respiratory tract infection and symptoms of wheezing in Shanghai. HBoV1 infection cases are often associated with other pathogens. The viral strains responsible for winter epidemics circulating among children in Shanghai belonged to the same genotype of HBoV1; thus, they may be derived from one common ancestor.
\end{abstract}

\section{Introduction}

Human bocavirus (HBoV) has been detected in both adult and children respiratory tract samples since its discovery in 2005 (1-3). The HBoV genome contains 3 open reading frames (ORFs), encoding 2 non-structural proteins (NS1 and NP1) and 2 viral capsid proteins (VP1 and VP2; VP2 overlaps with $V P 1$, but they have different start codes). $\mathrm{HBoV}$ strains with larger than $10 \%$ VPI nucleotide differences (VPD) were

Correspondence to: Dr Hui Yu, Department of Infectious Diseases, Children's Hospital of Fudan University, 399 Wanyuan Road, Minhang District, Shanghai 201102, P.R. China

E-mail: huiyuyuu@hotmail.com

Key words: human bocavirus, acute lower respiratory tract infection, children, genome analysis distinguished as different genotypes, while VPD between $5 \%$ and $10 \%$ was typing criteria for different subgenotypes. According to the typing criteria, the $\mathrm{HBoV}$ discovered in 2005 was termed HBoV1 $(4,5)$. Subsequently, 3 more genotypes of $\mathrm{HBoV}$ (HBoV2-4) were found from human excrement (4-6). $\mathrm{HBoV} 2$ can be divided into 2 subgenotypes, HBoV2A and HBoV2B. It has been shown that inter- and intra-genotype recombinations are present among the bocavirus (7).

In humans, the level of anti-HBoV1 antibody increases 2 months after birth and then undergoes a continuous decline before the age of 6 months. A continuous increase in antiHBoV1 antibody levels can be observed from the age of 6 months to 6 years. By the age of 2, approximately $80 \%$ of children have been infected by HBoV1-4 (8-10). Serological detection has revealed that $\mathrm{HBoV}$ has a long-term worldwide prevalence among individuals of all ages. Recent studies have indicated that HBoV1 can be cultivated in human respiratory epithelial cells in vitro $(11,12)$. However, no animal infection model has been established thus far; HBoV could not be confirmed as a human pathogen according to the Koch theory (13-15). Some reports have pointed out that HBoV1 is closely related to symptoms of wheezing in children $(8,16)$, while other studies have considered $\mathrm{HBoV} 1$ as a passerby or co-infector virus $(17,18)$. Evidence has shown that HBoV1-4 may also be involved in human gastrointestinal tract infections (1,19-21). In our previous study, HBoV1 was found to be closely associated with acute respiratory tract infection in children in Shanghai and most of the infected children had symptoms of wheezing (22). Whether HBoV2-4 is involved in acute respiratory tract infection with symptoms of wheezing is unknown and the genetic evolutionary relation of the epidemic HBoV1-4 strains has not been determined yet.

During the high-occurrence season for acute respiratory tract infections (wintertime, from December 2012 to February 2013), we collected samples of nasopharyngeal secretion (NPS) from hospitalized children with acute lower respiratory tract infection and symptoms of wheezing. We detected the existence of HBoV1-4 in the samples by nested PCR. The DNA sequences of $V P 1 / V P 2, N P 1$ and NS1 in the $\mathrm{HBoV}$-positive samples were further amplified, sequenced and aligned for phylogenetic tree construction. Our results revealed that the $\mathrm{HBoV}$ strains detected from the positive samples belonged to one genotype of $\mathrm{HBoV} 1$ and it was suggested that all the strains were derived from one common ancestor. 
Table I. The general nested PCR primer pairs for HBoV1-4 VP1/VP2 and HBoV1 NP1 and NS1.

\begin{tabular}{lll}
\hline Gene name & Primer name & \multicolumn{1}{c}{ Primer sequence } \\
\hline HBoV1-4 VP1 & AK-VP-F1 & 5'-CGCCGTGGCTCCTGCTCT-3' \\
HBoV1-4 VP2 & AK-VP-R1 & 5'-TGTTCGCCATCACAAAAGATGTG-3' \\
& AK-VP-F2 & 5'-GGCTCCTGCTCTAGGAAATAAAGAG-3' \\
HBoV1 NP1 & AK-VP-R2 & 5'-CCTGCTGTTAGGTCGTTGTTGTATGT-3' \\
& HBoV_2204F & 5'-GAGACATCGCAAGTGGACTAT-3' \\
& HBoV_3101R & 5'-TTGAGCAGCGCGATCAGCGTTA-3' \\
& HBoV_2321F & 5'-GCACAGCCACGTGACGAAGATGA-3' \\
HBoV1 NS1 & HBoV_3056R & 5'-GGATTAAATGGCCCAAGATA-3' \\
& Adel-OF & 5'-AGGTAAAACAAATATTGCAAAGGCCATAGTC-3' \\
& Adel-OR & 5'-TGGGAGTTCTCTCCGTCCGTATC-3' \\
& Adel-IF & 5'-AGGGTTTGTCTTTAACGATTGCAGACAAC-3' \\
& Adel-IR & 5'-TATACACAGAGTCGTCAGCACTATGAG-3'
\end{tabular}

\section{Materials and methods}

This study was approved by the Ethics Committee of the Children's Hospital of Fudan University, Shanghai, China. The 275 NPS samples were collected upon the agreement of the guardians of the infected children.

Materials. A total of 275 NPS samples were collected from children with symptoms of wheezing (within $48 \mathrm{~h}$ of hospitalization during the period between December 2012 and February 2013) with acute lower respiratory tract infection. The patients (158 males and 117 females) were aged between 1 month to 8 years; there were 56 cases of bronchitis and 219 cases of pneumonia (including 5 cases of severe pneumonia). All patients had symptoms of coughing and wheezing and a chest radiograph indicated the presence of bronchitis and pneumonia. Of the 275 patients, 136 had a fever $\left(>38^{\circ} \mathrm{C}\right)$. Patients displaying symptoms of coughing and wheezing after $48 \mathrm{~h}$ of hospitalization or with a past history of asthma were excluded from this study.

HBoVI-4 detection. Viral RNA was extracted according to the instructions provided with the Viral Genomic DNA/RNA Extraction kit [Cat. no. DP 315; Tiangen Biotech (Beijing) Co., Ltd., Beijing, China]. The general nested PCR primer pairs were designed for HBoV1-4 VPI/VP2 and HBoV1 NP1 $(1,4,23)$ (Table I). The available PCR reaction conditions and programs were used in our study $(1,4,6,23-25)$. The HBoV plasmid was kindly provided by Professor Zhou Rong from Guangzhou Medical University, Guangzhou, China.

Alignment analysis of HBoV1 VP1/VP2, NP1 and NS1 DNA sequences. The DNA sequences obtained were sequenced and aligned using online BLAST software. The DNA sequences were analyzed and phylogenetic trees were generated using MEGA5.1.

Detection of other common respiratory tract viruses and pathogens. The direct immune fluorescence technique (Respiratory Panel IFA Kit; Chemion) was used for antigen detection for respiratory syncytial virus (RSV), adenovirus (ADV), influenza virus (IFV)-A, IFV-B and parainfluenza virus (PIV)1-3. The fluorescent PCR and bacteria cultivation results of Mycoplasma pneumoniae and Chlamydia trachomatis for the 275 NPS samples were also obtained.

\section{Results}

Detection of HBoVI-4. The nested PCR results revealed that 15 of the 275 NPS samples were HBoV1-positive; the detection rate was $5.45 \%$. With the use of common primers for HBoV1-4 $V P 1 / V P 2, \mathrm{HBoV} 1 \mathrm{NP} 1$ and HBoV1 NS1, 15, 15, and 8 positive samples were detected, respectively, by nested PCR. Seven samples with positive signals of $V P 1 / V P 2$ and $N P 1$ failed to yield nested RT-PCR products using $N S 1$ primers. The clinical information of the $15 \mathrm{HBoV1}$-positive samples is summarized in Table II.

All the PCR products obtained were sequenced. All the sequences were pooled together with the HBoV1 whole genome (available in GeneBank) for homology analysis using ClustalW (MEGA5.1). The detailed homology information for $V P 1 / V P 2, N P 1$ and $N S 1$ is shown in Figs. 1-3, respectively.

Sequence homology analysis of the HBoVI VPI/VP2 gene. The $15 \mathrm{HBoV} 1$ strains in our study showed a maximum homology difference of $1.7 \%$ with previously discovered strains [strains reported in 2010 and 2011 in Guangzhou: GZ2010-1(JN128956), GZ2010-03(JN128953), GZ2011-01(JN128954) and GZ201104(JN128955); strains reported in 2012 in Guangzhou: GZ4785(JN794565) and GZ9081(JN794566); strains reported in 2012 in the US: KU3(JQ411251)]. A minimum difference of $28.1 \%$ was shown between the 2009 Australian HBoV2 strains [W298(FJ948860) and W208(EU082214)] and the newly discovered strains. A minimum difference of $30.8 \%$ was shown between the 2009 Australian HBoV3(NC_012564) and the new HBoV1 strains. A minimum difference of $30.6 \%$ was shown between the 2010 American HBoV4(NC_012729) and the new HBoV1 strains. The maximum difference among the 15 new $\mathrm{HBoV} 1$ strains was $1.3 \%$, which indicated that they belonged to the same genotype (Fig. 1). 
Table II. Clinical information of children infected by HBoV1.

\begin{tabular}{|c|c|c|c|c|c|}
\hline No. & Age & Gender & Clinical diagnosis & Diarrhea & Co-infected pathogens \\
\hline 1 & 3 Months & Female & Pneumonia & Yes & RSV \\
\hline 2 & 11 Months & Male & Pneumonia & Yes & RSV, hMPV \\
\hline 3 & 3 Years & Male & Bronchitis & No & $\mathrm{Hi}$ \\
\hline 4 & 2 Years & Female & Pneumonia & Yes & \\
\hline 5 & 3 Years & Female & Pneumonia & No & $\mathrm{ADV}, \mathrm{MC}$ \\
\hline 6 & 8 Months & Female & Bronchitis & Yes & RSV \\
\hline 7 & 6 Years & Male & Pneumonia & No & MP \\
\hline 8 & 2 Years & Male & Bronchitis & No & RSV \\
\hline 9 & 8 Years & Male & Pneumonia & No & MP, Strep \\
\hline 10 & 11 Months & Male & Pneumonia & No & RSV, Hi \\
\hline 11 & 2 Years & Female & Pneumonia & Yes & IAV \\
\hline 12 & 8 Months & Male & Bronchitis & Yes & \\
\hline 13 & 3 Years & Female & Pneumonia & No & RSV \\
\hline 14 & 4 Years & Female & Pneumonia & No & $\mathrm{ADV}$ \\
\hline 15 & 2 Years & Male & Pneumonia & Yes & \\
\hline
\end{tabular}

RSV, respiratory syncytial virus; hMPV, human metapneumovirus; ADV, adenovirus; IAV, influenza A virus; Hi, Haemophilus influenzae; MC, Moraxella catarrhalis; Strep, Streptococcus pneumoniae; MP, Mycoplasma pneumoniae.

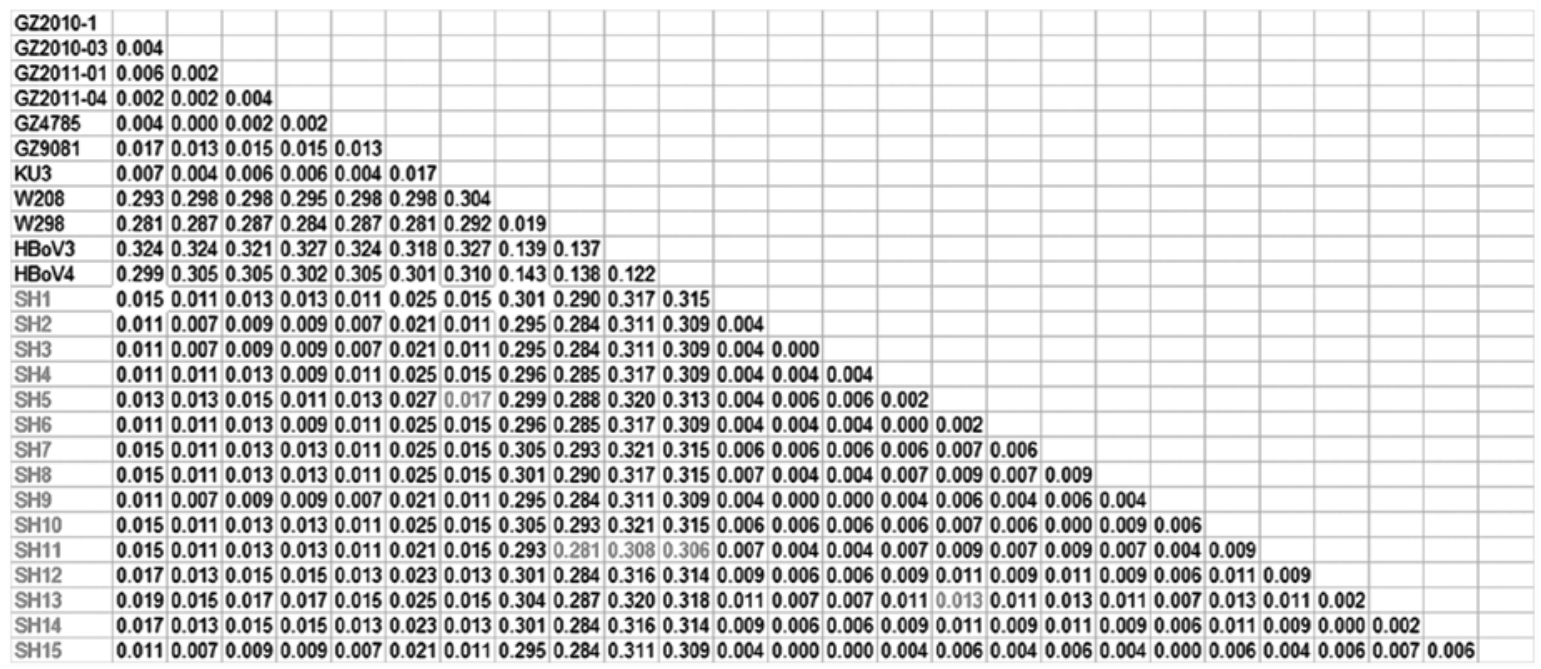

Figure 1. Sequence homology of $V P 1 / V P 2$ for the $15 \mathrm{HBoV1}$ strains and whole genome sequence of HBoV1 (available in GenBank). ClustalW (MEGA5.1) was used for sequence homology comparison and calculation.

Sequence homology analysis of the HBoVl NPl gene. The homology differences among the newly discovered HBoV strains and previously discovered strains were also calculated based on the NPl sequences. A maximum difference in homology of $2.3 \%$ was observed between the $15 \mathrm{HBoV} 1$ strains and the previously discovered strains (GZ2010-1, GZ2010-03, GZ2011-01, GZ2011-04, GZ4785, GZ9081 and KU3). The minimum difference between W298 and W208 and the newly discovered HBoV1 strains was $27.8 \%$; the minimum difference between the new strains and $\mathrm{HBOV} 3$ was $15.2 \%$; the minimum difference between the new strains and $\mathrm{HBoV} 4$ was $27.3 \%$; the maximum difference between the 15 new HBoV1 strains was $1.8 \%$, which indicated that they belonged to the same genotype (Fig. 2).

Sequence homology analysis of the HBoVl NS1 gene. The homology differences among the newly discovered HBoV strains and the previously discovered strains were also calculated based on the $8 \mathrm{NS} 1$ sequences. A maximum difference in homology of $3.6 \%$ was observed between the $8 \mathrm{HBoV} 1$ strains and the previously discovered strains (GZ2010-1, GZ2010-03, GZ2011-01, GZ2011-04, GZ4785, GZ9081 and KU3). A minimum difference of $19.5 \%$ was shown among the new strains and W298 


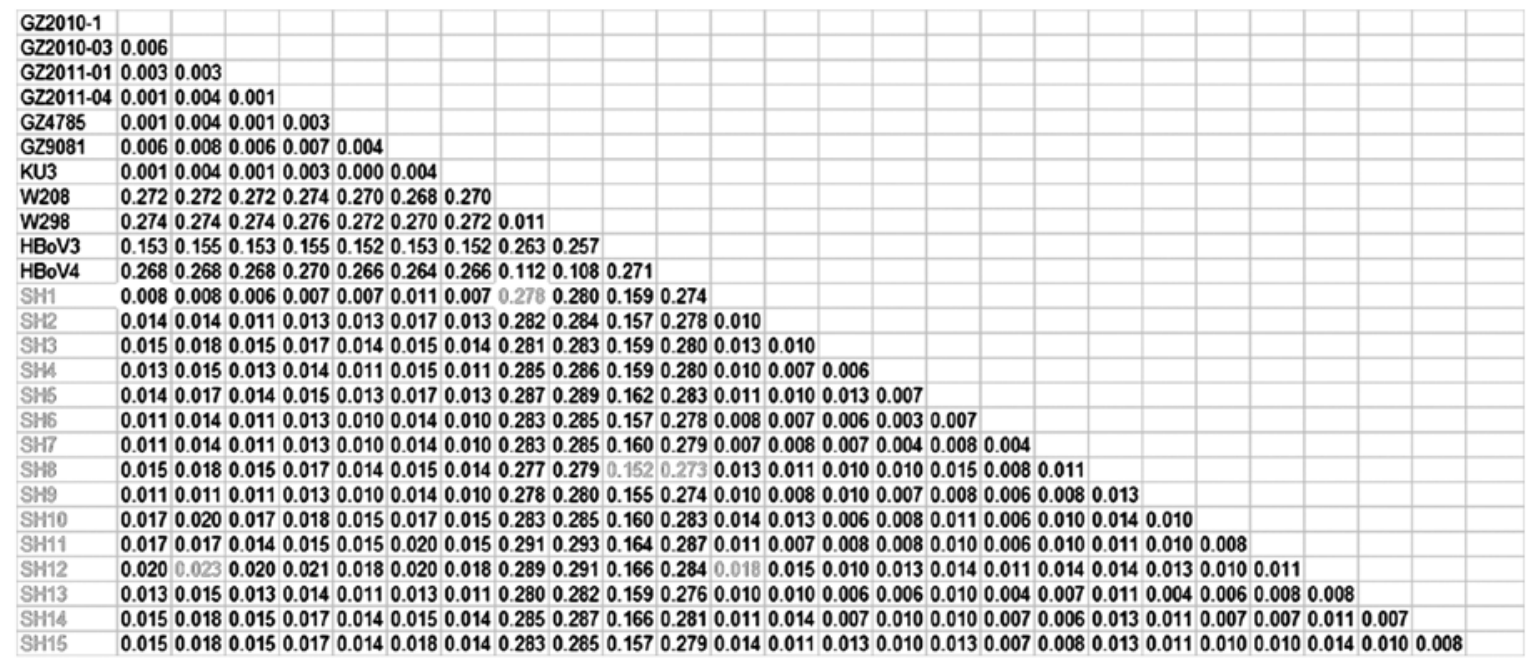

Figure 2. Sequence homology of NP1 for the $15 \mathrm{HBoV} 1$ strains and whole genome sequence of HBoV1 (available in GenBank). ClustalW (MEGA5.1) was used for sequence homology comparison and calculation.

\begin{tabular}{|c|c|c|c|c|c|c|c|c|c|c|c|c|c|c|c|c|c|c|}
\hline \multirow{3}{*}{$\begin{array}{l}\text { GZ2010-1 } \\
\text { GZ2010-03 }\end{array}$} & & & & & & & & & & & & & & & & & & \\
\hline & 0.010 & & & & & & & & & & & & & & & & & \\
\hline & 0.008 & 0.014 & & & & & & & & & & & & & & & & \\
\hline GZ2011-04 & 0.008 & 0.014 & 0.000 & & & & & & & & & & & & & & & \\
\hline GZ4785 & 0.008 & 0.014 & 0.000 & 0.000 & & & & & & & & & & & & & & \\
\hline GZ9081 & 0.008 & 0.014 & 0.000 & 0.000 & 0.000 & & & & & & & & & & & & & \\
\hline KU3 & 0.008 & 0.014 & 0.000 & 0.000 & 0.000 & 0.000 & & & & & & & & & & & & \\
\hline W208 & 0.208 & 0.211 & 0.205 & 0.205 & 0.205 & 0.205 & 0.205 & & & & & & & & & & & \\
\hline W298 & 0.211 & 0.213 & 0.207 & 0.207 & 0.207 & 0.207 & 0.207 & 0.006 & & & & & & & & & & \\
\hline HBoV3 & 0.102 & 0.108 & 0.092 & 0.092 & 0.092 & 0.092 & 0.092 & 0.182 & 0.185 & & & & & & & & & \\
\hline HBoV4 & 0.192 & 0.200 & 0.189 & 0.189 & 0.189 & 0.189 & 0.189 & 0.083 & 0.090 & 0.182 & & & & & & & & \\
\hline SH1 & 0.027 & 0.033 & 0.019 & 0.019 & 0.019 & 0.019 & 0.019 & 0.195 & 0.198 & 0.097 & 0.192 & & & & & & & \\
\hline $\mathrm{SH} 3$ & 0.028 & 0.034 & 0.020 & 0.020 & 0.020 & 0.020 & 0.020 & 0.196 & 0.198 & 0.097 & 0.192 & 0.002 & & & & & & \\
\hline $\mathrm{SH} 4$ & 0.027 & 0.033 & 0.019 & 0.019 & 0.019 & 0.019 & 0.019 & 0.195 & 0.198 & 0.097 & 0.187 & 0.006 & 0.006 & & & & & \\
\hline SH6 & 0.029 & 0.035 & 0.021 & 0.021 & 0.021 & 0.021 & 0.021 & 0.198 & 0.200 & 0.099 & 0.189 & 0.006 & 0.004 & 0.002 & & & & \\
\hline $\mathrm{SH} 7$ & 0.030 & 0.036 & 0.021 & 0.021 & 0.021 & 0.021 & 0.021 & 0.198 & 0.201 & 0.099 & 0.190 & 0.004 & 0.002 & 0.004 & 0.002 & & & \\
\hline SH8 & 0.031 & 0.038 & 0.023 & 0.023 & 0.023 & 0.023 & 0.023 & 0.200 & 0.203 & 0.101 & 0.192 & 0.008 & 0.006 & 0.004 & 0.002 & 0.004 & & \\
\hline SH14 & 0.027 & 0.033 & 0.019 & 0.019 & 0.019 & 0.019 & 0.019 & 0.195 & 0.198 & 0.097 & 0.187 & 0.006 & 0.006 & 0.000 & 0.002 & 0.004 & 0.004 & \\
\hline SH15 & 0.036 & 0.042 & 0.027 & 0.027 & 0.027 & 0.027 & 0.027 & 0.213 & 0.216 & 0.108 & 0.200 & 0.014 & 0.016 & 0.020 & 0.020 & 0.018 & 0.021 & 0.020 \\
\hline
\end{tabular}

Figure 3. Sequence homology of NS1 for the $8 \mathrm{HBoV} 1$ strains and whole genome sequence of HBoV1 (available in GenBank). ClustalW (MEGA5.1) was used for sequence homology comparison and calculation.

and W208; the minimum difference between the new strains and $\mathrm{HBoV} 3$ was $9.7 \%$; the minimum difference between the new strains and HBoV4 was $18.7 \%$. The maximum difference between the 8 new HBoV1 strains was $2.1 \%$, which indicated that they belonged to the same genotype (Fig. 3). The $8 \mathrm{HBoV} 1$ strains showed a minimum difference of $10 \%$ with HBoV3, which supported the assumption that $\mathrm{HBoV} 3$ was derived from the recombination of $\mathrm{HBoV} 1$ and $\mathrm{HBoV} 2(4,25,26)$.

Phylogenetic tree generation for the HBoVl gene. The positive PCR products of $15 \mathrm{VPl} / \mathrm{VP} 2$ genes, $15 \mathrm{NP} 1$ genes and 8 NS1 genes were pooled for phylogenetic tree construction (Fig. 4). All 3 phylogenetic trees showed that the 15 detected viral strains were scattered on the same branch, which indicated that they belonged to the same HBoV1 genotype.

Detection of other common pathogens. The detection rates for 8 common viruses [RSV, ADV, IFA/B, PIV1-3 and human metapneumovirus (hMPV)] were 44.73, 2.18, 1.45, 1.45 and $3.64 \%$, respectively. The total detection rate was $53.4 \%$ (147/275); RSV was the most common pathogen (44.73\%) and the detection rate of HBoV1 $(5.45 \%)$ was second to RSV. The 15 HBoV1-positive samples were co-detected with 6 cases of RSV and 2 cases of hMPV. Thirteen cases of Mycoplasma pneumoniae and Chlamydia trachomatis were identified by qRT-PCR (4.73\%); the positive rate of the bacterial culture was $24.36 \%$ (67/275); the co-infection rate was $16.0 \%$ (44/275); the total detection rate of all the pathogens was $72.0 \%(198 / 275)$.

\section{Discussion}

$\mathrm{HBoV}$ is a newly discovered Parvoviridae virus. HBoV particles can be detected in respiratory secretions and digestive excrements. A number of scientists have considered that $\mathrm{HBoV}$ is closely connected with human respiratory tract infections. However, there is still controversy as to the involvement of HBoV1-4 in children with symptoms of wheezing. The detection of $\mathrm{HBoV}$ DNA or particles in the secretions of patients 

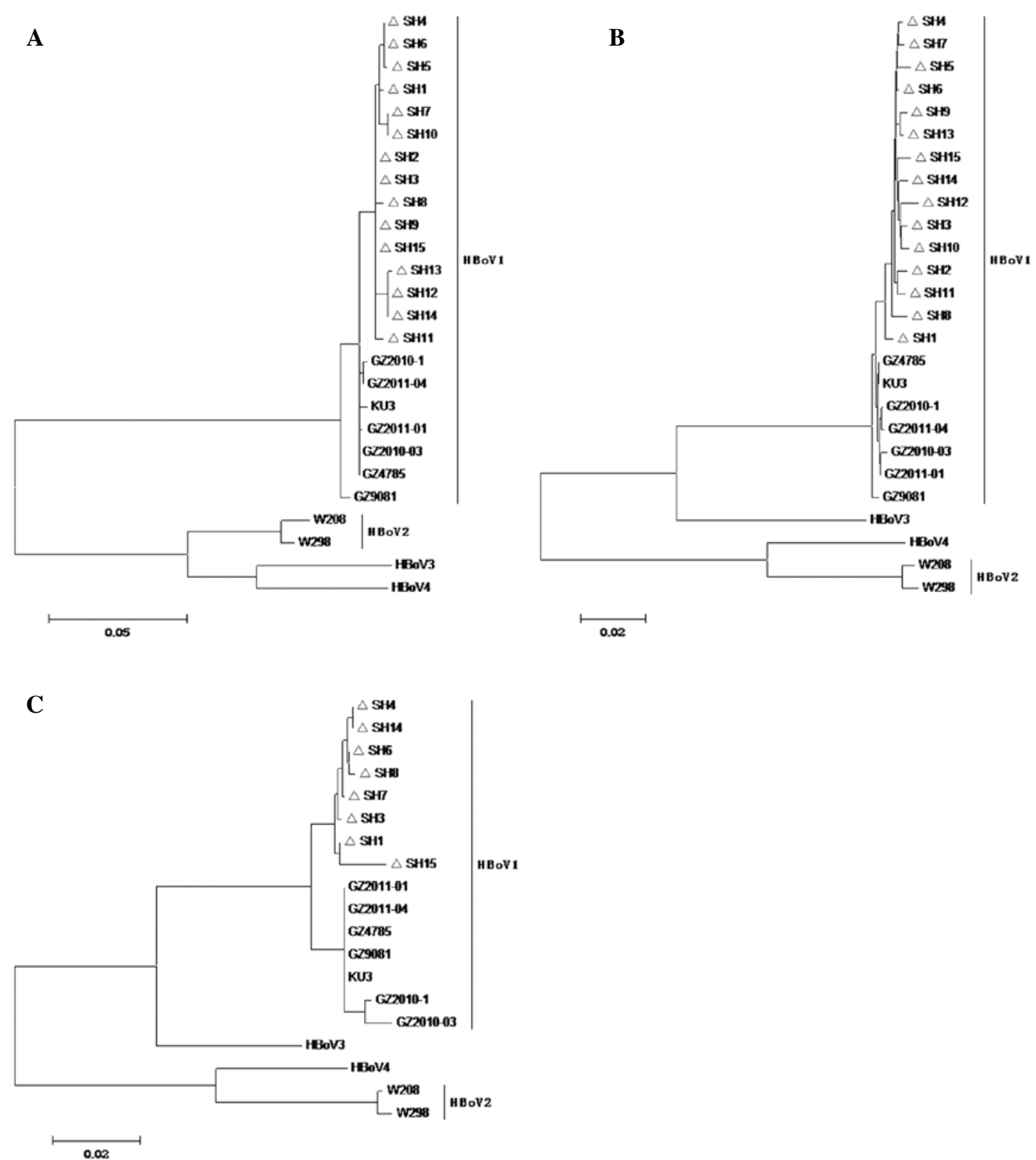

Figure 4. Phylogenetic tree based on nucleotide sequences of VP1/VP2, NP1 and NS1 in the 15 detected HBoV1 strains. (A) Phylogenetic tree for VP1/VP2; (B) phylogenetic tree for NP1; (C) phylogenetic tree for NS1. Phylogenetic analysis was conducted using MEGA5.1 (www.megasoftware.com). Genetic distances were calculated with the pairwise distance method. Phylogenetic trees were constructed with the neighbor-joining method.

with symptoms of wheezing and the positive transition of serological HBoV antibody were used in this study as alternative methods of examining the infections, although no animal model was available for an $\mathrm{HBoV}$ infection study.

We discovered $15 \mathrm{HBoV1-positive} \mathrm{samples} \mathrm{from} \mathrm{the}$ 275 NPS collected from hospitalized children with acute lower respiratory tract infection with symptoms of wheezing; the detection rate was $5.45 \%$, which was second to RSV. This indicates that HBoV1 may be one of the common pathogens responsible for the hospitalization of children with acute lower respiratory tract infection with symptoms of wheezing in Shanghai. As we did not include a control group with no symptoms, no serological and viremia detection were perrormed in our study; the $15 \mathrm{HBoV} 1$ strains may be co-pathogens only or just viruses being carried by the patients. No HBoV2-4 signals were detected, which indicated that HBoV2-4 could not be the pathogens responsible for the hospitalization of children with acute lower respiratory tract infection with symptoms of wheezing in the winter of 2012; this is consistent with the low detection rate of $\mathrm{HBoV} 2-4$ in respiratory tract secretions (27-29). Although 7 HBoV1-positive cases displayed symptoms of diarrhea, it could not be confirmed that the digestive tract removed the viral particles, as no excrement samples of the HBoV1-positive cases were collected and detected. Further studies are required to fully investigate this issue.

The homology differences among the newly discovered $\mathrm{HBoV}$ strains and the previously discovered strains were calculated based on the $V P 1 / V P 2, N P 1$ and NS1 sequences. All the results revealed that the 15 new HBoV1 strains belonged to the same genotype (Figs. 1-3). All the 15 strains were scattered on the same branch in the 3 phylogenetic trees (Fig. 4). This indicated that the 15 new HBoV1 strains were very likely derived 
from one common $\mathrm{HBoV} 1$ ancestor with pathogenicity, which underwent evolution through inter-genotype recombination.

The discovery of HBoV1-4 indicated that HBoV has a feature of genetic diversities. Being consistent with a recent report (29), the analysis of whole genome sequences in GenBank revealed that the gene recombinations occurred not only among HBoV1-4, but also among the members in the same genotypes. The analysis of different ORFs led to the same conclusion that all the newly discovered HBoV1 strains belonged to the same genotype. Further persistent monitoring is required to determine whether the HBoV1 genotype is closely related with digestive tract infections in children with symptoms of wheezing in Shanghai.

In our study, only HBoV1 was detected by nested PCR from the 275 NPS samples collected from the hospitalized children with symptoms of wheezing in the winter of 2012. The detection rate of HBoV1 was 5.45\% (15/275), which was second only to RSV. HBoV1 had a high co-infection rate with other potential pathogens in most of the samples. The 15 newly discovered HBoV1 strains belonged to the same subgroup and thus may be derived from one common ancestor. Our results indicated that HBoV1 may be one of the common pathogens responsible for the hospitalization of children with acute lower respiratory tract infection with symptoms of wheezing in Shanghai.

\section{Acknowledgements}

This study was supported by a grant from the Key Discipline Construction of Public Health in Shanghai (08GWZX0102).

\section{References}

1. Khamrin $\mathrm{P}$, Malasao R, Chaimongkol N, et al: Circulating of human bocavirus 1,2,3, and 4 in pediatric patients with acute gastroenteritis in Thailand. Infect Genet and Evol 12: 565-569, 2012.

2. Liu WK, Chen DH, Liu Q, et al: Detection of human bocavirus from children and adults with acute respiratory tract illness in Guangzhou, southern China. BMC Infect Dis 11: 345, 2011.

3. Guo L, Wang Y, Zhou H, et al: Differential seroprevalence of human bocavirus species 1-4 in Beijing, China. PLoS One 7: e39644, 2012.

4. Kapoor A, Simmonds P, Slikas E, et al: Human bocaviruses are highly diverse, dispersed, recombination prone, and prevalent in enteric infections. J Infect Dis 201: 1633-1643, 2010.

5. Kapoor A, Slikas E, Simmonds P, et al: A newly identified bocavirus species in human stool. J Infect Dis 199: 196-200, 2009.

6. Arthur JL, Higgins GD, Davidson GP, Givney RC and Ratcliff RM: A novel bocavirus associated with acute gastroenteritis in Australian children. PLoS Pathog 5: e1000391, 2009.

7. Fu X, Wang X, Ni B, et al: Recombination analysis based on the complete genome of bocavirus. Virology J 8: 182, 2011.

8. Kantola K, Hedman L, Arthur J, et al: Seroepidemiology of human bocaviruses 1-4. J Infect Dis 204: 1403-1412, 2011.
9. Endo R, Ishiguro N, Kikuta H, et al: Seroepidemiology of human bocavirus in Hokkaido prefecture, Japan. J Clin Microbiol 45: 3218-3223, 2007

10. Hustedt JW, Christie C, Hustedt MM, Esposito D and Vazquez M: Seroepidemiology of human bocavirus infection in Jamaica. PLoS One 7: e38206, 2012.

11. Deng X, Yan Z, Luo Y, et al: In vitro modeling of human bocavirus 1 infection of polarized primary human airway epithelia. J Virol 87: 4097-4102, 2013.

12. Huang Q, Deng X, Yan Z, et al: Establishment of a reverse genetics system for studying human bocavirus in human airway epithelia. PLoS Pathog 8: e1002899, 2012.

13. Rivers TM: Viruses and Koch's Postulates. J Bacteriol 33: 1-12, 1937.

14. Weissbrich B, Neske F, Schubert J, et al: Frequent detection of bocavirus DNA in German children with respiratory tract infections. BMC Infect Dis 6: 109, 2006.

15. Lusebrink J, Schildgen V, Tillmann RL, et al: Detection of headto-tail DNA sequences of human bocavirus in clinical samples. PLoS One 6: e19457, 2011.

16. Deng Y, Gu X, Zhao X, et al: High viral load of human bocavirus correlates with duration of wheezing in children with severe lower respiratory tract infection. PLoS One 7: e34353, 2012.

17. Longtin J, Gubbay JB, Patel S and Low DE: High prevalence of asymptomatic bocavirus in daycare: is otitis media a confounder? J Infect Dis 202: 1617, 2010.

18. Debiaggi M, Canducci F, Ceresola ER and Clementi M: The role of infections and coinfections with newly identified and emerging respiratory viruses in children. Virol J 9: 247, 2012.

19. Proenca-Modena J, Martinez M, Amarilla A, et al: Viral load of human bocavirus-1 in stools from children with viral diarrhoea in Paraguay. Epidemiol Infect: Feb 21, 2013 (Epub ahead of print).

20. Risku M, Kätkä M, Lappalainen S, Räsänen S and Vesikari T: Human bocavirus types 1,2 and 3 in acute gastroenteritis of childhood. Acta Paediatr 101: e405-e410, 2012.

21. Khamrin P, Thongprachum A, Shimizu H, et al: Detection of human bocavirus 1 and 2 from children with acute gastroenteritis in Japan. J Med Virol 84: 901-905, 2012.

22. Zeng M, Zhu QR, Wang XH, Yu H and Shen J: Human bocavirus in children with respiratory tract infection in Shanghai: a retrospective study. World J Pediatr 6: 65-70, 2010.

23. Mitui MT, Tabib SM, Matsumoto T, et al: Detection of human bocavirus in the cerebrospinal fluid of children with encephalitis. Clin Infect Dis 54: 964-967, 2012.

24. Ghietto LM, Cámara A, Zhou Y, et al: High prevalence of human bocavirus 1 in infants with lower acute respiratory tract disease in Argentina, 2007-2009. Braz J Infect Dis 16: 38-44, 2012.

25. Cheng W, Chen J, Xu Z, et al: Phylogenetic and recombination analysis of human bocavirus 2. BMC Infect Dis 11: 50, 2011.

26. Chieochansin T, Simmonds P and Poovorawan Y: Determination and analysis of complete coding sequence regions of new discovered human bocavirus types 2 and 3. Arch Virol 155: 2023-2028, 2010.

27. Koseki N, Teramoto S, Kaiho M, et al: Detection of human bocaviruses 1 to 4 from nasopharyngeal swab samples collected from patients with respiratory tract infections. J Clin Microbiol 50: 2118-2121, 2012

28. Xu L, He X, Zhang DM, et al: Surveillance and genome analysis of human bocavirus in patients with respiratory infection in Guangzhou, China. PLoS One 7: e44876, 2012.

29. Abdel-Moneim AS, Kamel MM, Al-Ghamdi AS and Al-Malky MI: Detection of bocavirus in children suffering from acute respiratory tract infections in Saudi Arabia. PLoS One 8: e55500, 2013 